\title{
Investigating the significance of parameters affecting the defects in HPDC component using design of experiment method.
}

\author{
Dasaratha Prabhu B ${ }^{1}$, Ramesh Babu $\mathrm{K}^{2}$ \\ '(Department of PG studies, GT\&TC, Mysore, Visvesvaraya technological University, India.) \\ ${ }_{2}^{2}$ (Principal of PG studies, GT\&tc, Mysore, Visvesvaraya technological University, India.)
}

\begin{abstract}
A sample lead seal is being prepared in a HPDC D250 machine. In which the experiment is being done by collecting the various parameters affecting the causes for a defect in a component, Where the affecting parameters are pouring temperature ranging from $(400-450)^{\circ} \mathrm{C}$, tool temperature $(80-110){ }^{\circ} \mathrm{C}$, pouring time (1.8-4.5) seconds and cooling medium (air and oil). This experiment was conducted at room temperature; the data's have been collected at various trails and being interrupted by statistical analysis software to find the significance of various parameters affecting the defect in the component. Design of experiment (DOE) by Taguchi method is being conducted by generating an orthogonal array. A response for defect is being input into the worksheet and analysis is being carried out. The signal-to-noise ratio graph shows you the various parameters affecting in a each defect such as blowhole, bubble and flash defect .Taguchi method is evident to conclude that major significance is caused by pouring Temperature for blowhole defect, pouring time influences the major bubble defect in a component and the flash defect is being signified by major parameters like tool temperature and cooling medium.
\end{abstract}

Keywords- high pressure die casting, blowhole, bubble and flash.

\section{Introduction}

Die casting are the highest volume, mass produced item manufactured by the metal work industry and it is one of the fastest and most effective methods for producing a wide range of component of products ranging from small to large size. Die casting is similar to mold casting except the metal is injected into the mold under high pressure. This results in uniform part and eliminate the post machining and time saving. Lead alloys are being used to produce lead sealing for the meter board. Lead has the advantages of low melting temperature and extreme malleability, which allow easy casting, shaping and joining of lead articles, besides this lead is slow to corrode. However, compared with other metals, lead has extremely low strength. Lead is rarely used in its pure form. The very high density of lead lends itself to some quite different electrical application.

Die casting process allows us to produce high production of simple or complex components in manufacturing technology. While casting a product in the machine we come to arise different defects, which is being produced in turn reduces the quality of the product .The defect which is being found while conducting the production work in high pressure die casting component such as Blowhole, bubble and flash. We have collected the data which is being a major cause for the defect produce in a component such as the effecting parameters like pouring temperature ranging from $(400,450)$, pouring time ranging from $(1.8$ to 4.5$)$ seconds, tool temperature ranging from (80to 110) and cooling medium (air, air+oil). This has given an impetus to industrial production of parameters on defects. For example, interfacial heat-transfer coefficients during the solidification of Al-Si alloys against coated die steel chills with varying chill temperature, coating thickness and coating type [1-7]. C.P. Hallam and W.D. Griffiths [8] measured interfacial heat-transfer coefficients during the solidification of Al-Si alloys against coated die steel chills with varying chill temperature, coating thickness and coating type. Guo Zhi-peng et al. [9] focuses on the determination of the interfacial heat transfer coefficient (IHTC) at the metal-die interface in the high pressure die casting (HPDC) process. PENG et al. [10] investigated the effects of process parameters, casting thickness, and alloys on the metal-die interfacial heat-transfer coefficient (IHTC) in the HPDC process. Zhipeng et al. [11] The interfacial heat transfer between metal and die during high pressure die casting. Persson et al. [12] Experimentally evaluated the temperature variations in the surface layer of hot work tool steel during actual brass die casting. Micowski Teufert [13 Presented results of an investigation of methods of controlling flash and the applications of a real-time closed-loop shot control system to control impact pressure. The effects of process variables on the quality of high-pressure die cast components with the aid of in-cavity pressure sensors[14]. Chiang, Liu \& Tsai [15] Proposed mathematical models for the modeling and analysis of the effects of machining parameters on the performance characteristics in the HPDC process of Al-SI alloys which were developed using the response surface methodology. Jerald Brevick et al[16] Conducted a literature review regarding energy used in die-casting, and then create and distribute a survey regarding energy consumption to North American Die Casting Association. Investigated the effect of control of cooling, the average cooling rate and the effect of heat released [17-19]. Q. HAN and S. VISWANATHAN [20] proposed a 
mechanism of soldering of an aluminum alloy die casting to a steel die. Sumanth Shankar and Diran Apelian [21] provided a comprehensive understanding of the reactions at the ferrous die/molten metal interface in a metal mold casting operation. Hangai and Utsunomiya [22] fabricated a closed-cell porous aluminum using gases intrinsically contained in aluminum alloy die castings without using a blowing agent. Sabau and Viswanathan[23] developed a comprehensive methodology that takes into account solidification, shrinkagedriven interdendritic fluid flow, hydrogen precipitation, and porosity evolution for the prediction of the micro porosity fraction and distribution in aluminum alloy castings. ZHU et al. [24] developed a numerical model for predicting micro porosity formation in aluminum castings. Since these various parameters play a important role in defects investigation.

Design of experiments (DOE) using Taguchi approach is a standardized form of experimental technique. DOE is an experimental strategy in which effects of multiple factor are studied simultaneously by running test at various levels of the factor. Glen [25] found that the Taguchi approach to experimentation provides an orderly way to collect, analyze, and interpret data to satisfy the objective of study. By using these methods in design of experiments, one can obtain the maximum amount of information for the amount of experiments used. This is accomplished by the efficient use of experiments runs to the combination of variables studied. Taguchi [26] proposed that the most important stage in the plan of experiments is selection of factors. Taguchi technique creates a standard orthogonal array to accommodate the effect of several factors on the target value and defines the plan of experiments.The experimental results are analyzed using analysis of means and variance to study the influence of factor. To evaluate the possibilities to identify the significance of the factor which is being influence on the defect response. Further, Taguchi experimental design approach is applied to identify the significance and to carry out the interactions predominantly influencing the defect in HPDC component.

\subsection{Experimental Details.}

The lead seal samples are being produced in high pressure die casting machine D250 model under a injection pressure of around 5 tons, which is kept constant throughout the experiment. And the varying operating parameters like pouring temperature (400 to 450$){ }^{\circ} \mathrm{c}$, cooling medium used is air as well as oil, tool temperature ( 80 to 110$)^{\circ} \mathrm{c}$ and pouring time (1.8 to 4.5$)$ seconds.

\subsubsection{Details Of The Component.}

- Material of the component : Pure Lead

- Weight: $25 \mathrm{~kg} /$ ingot

- Dimensions: $\varnothing 5 * 2 \mathrm{~cm}$

- Chemical Composition: sb. as. sn. cu. bi. fe. ni. ag. zn, ca, s, al, pb.

- $\quad \mathrm{Pb}($ Min): 99.94

- Alloy type: non alloy.

- Specifications Pure lead ingot-Purity: over $99.97 \%$

\subsubsection{Lead Seal Defect Test.}

Lead seal component test were conducted in D250 HPDC machine under room temperature. The details of the defect test procedure describe elsewhere [27]. The sample was being produced by pouring a molten metal in the shot sleeve, where the pouring time is being noted in a stop watch and the pouring temperature is being noted in the thermocouple. Similarly for tool temperature values are being noted and cooling medium used in the process such as air and oil. After the production of lead seal which is being removed from the mold are carried for observation. To identify the number of defects produced in a cycle and that defects consist of different defects such as blowhole, bubble and flash. By noting down the different value of each defect produce, by conducting at least 2 test and the average value obtained is being used in the analysis .The experiment were carried out in HPDC machine, where the defects in each trail is observed and noted, and value are being interact in mini tab software.

Where pouring temperature in ${ }^{\circ} \mathrm{C}$, pouring time in seconds and tool temp in ${ }^{\circ} \mathrm{C}$.

\section{Design Of Experiment.}

Design of experiments (DOE) using Taguchi approach is a standardized form of experimental design technique .DOE is an experimental strategy in which effects of multiple factors are studied simultaneously by running test at various levels of the factor. Factor is a variable or a parameter that has a direct influence on the output. Levels are the description that define the conditions of the factor held while running the experiment .Design of experiment is a powerful analysis tool for modeling and analyzing the influence of control factors on performance output .The defect test are carried out under operating condition given in the Table 1. 


\begin{tabular}{|lccc|}
\hline FACTORS & LEVEL 1 & LEVEL 2 \\
\hline $\begin{array}{l}\text { POURING } \\
\text { TEMP }\end{array}$ & & 400 & 450 \\
\hline POURING & & 1.8 & 4.5 \\
TIME & & & AIR+OIL \\
\hline COOLING & AIR & & 110 \\
\hline TOOL TEMP & & 80 & \\
\hline
\end{tabular}

Table1. Operating conditions.

The tests are conducted at room temperature as per experimental design. Taguchi design of experiment methods are used to optimize the experimental design based on the number of control factors and the number of levels. Two orthogonal array designs L16 and L32 can be used. In this present work the L16 is used as the experimental design.

The experimental observations are transformed into signal-to-noise ratio $(\mathrm{S} / \mathrm{N})$ ratios. There are several $\mathrm{S} / \mathrm{N}$ ratios available depending on the type of characteristics. The $\mathrm{S} / \mathrm{N}$ for minimum defect rate coming under small is better characteristics, which can be calculated as logarithmic transformation of the function as shown below.

$$
\mathrm{SN}=-10 \log \left(\sum_{\mathrm{u}=1}^{\mathrm{Ni}}\left(\mathrm{y}_{\mathrm{u}}^{2} / \mathrm{N}_{\mathrm{i}}\right)\right)
$$

Where $\mathrm{i}$ is the experimental number, $\mathrm{u}$ is the trail number and $\mathrm{N}_{\mathrm{i}}$ is the number of trials for experiment $\mathrm{i}$. "Lower is better" (LB) characteristic, with the above $\mathrm{S} / \mathrm{N}$ transformation, is suitable for minimization of defect.

\subsection{Defects In A Component.}

\section{Reults And Discussion}

While carrying an experiment the common defects found while ejecting a component from the mold was blowhole, this defect is caused due to the temperature variation in the tool. Bubble, this defect is caused due to air entraps in the mold formation to bubble like blister formation. Flash, this defect is caused over the parting surface of the component. And the defects which are formed are shown in the figure1.
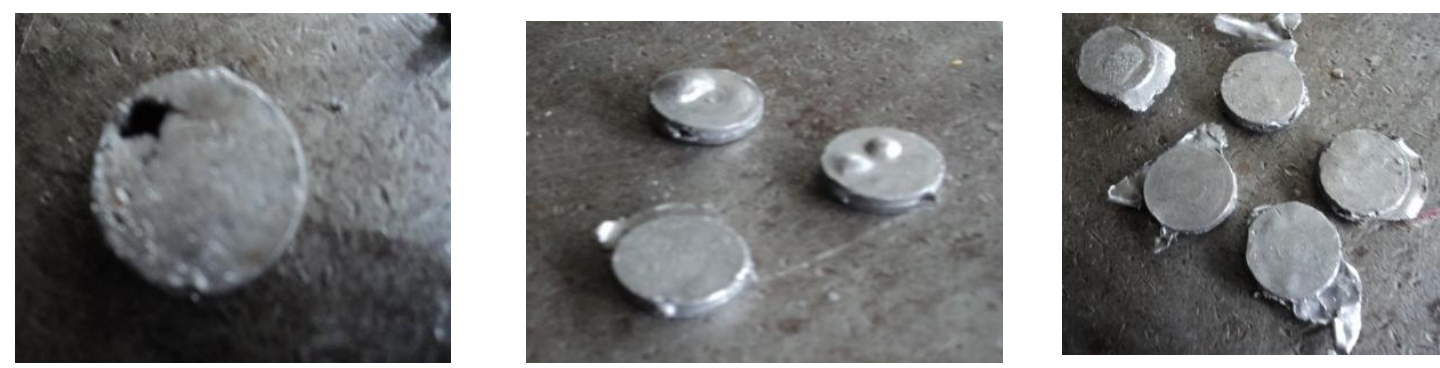

Figure1.defects.

\subsection{DATA INPUT.}

\begin{tabular}{|c|c|c|c|c|c|c|c|c|c|c|c|c|c|c|c|c|c|c|c|c|}
\hline Experiments & PTM & PT & CM & TTM & BH 1ST & BH 2ND & Experiments & PTM & PT & CM & TTM & BB 1ST & $\mathrm{BB} 2 \mathrm{ND}$ & Experiments & PTM & PT & $C M$ & $\pi M$ & FL1ST & $\overline{F L 2 N D}$ \\
\hline 1 & 400 & 1.8 & A & 80 & 12 & 13 & 1 & 400 & 1.8 & A & 80 & 10 & 7 & 1 & 400 & 1.8 & A & 80 & 5 & 5 \\
\hline 2 & 400 & 1.8 & A & 110 & 15 & 12 & 2 & 400 & 1.8 & A & 110 & 7 & 9 & 2 & 400 & 18 & A & 110 & 3 & 2 \\
\hline 3 & 400 & 4.5 & $A O$ & 80 & 6 & 5 & 3 & 400 & 4.5 & AO & 80 & 6 & 5 & 3 & 400 & 4.5 & AO & 80 & 4 & 1 \\
\hline 4 & 400 & 4.5 & $A O$ & 110 & 6 & 9 & 4 & 400 & 4.5 & AO & 110 & 7 & 7 & 4 & 400 & 4.5 & AO & 110 & 4 & 3 \\
\hline 5 & 400 & 1.8 & $A O$ & 80 & 12 & 14 & 5 & 400 & 1.8 & AO & 80 & 7 & 4 & 5 & 400 & 18 & AO & 80 & 3 & 5 \\
\hline 6 & 400 & 1.8 & $A O$ & 110 & 13 & 11 & 6 & 400 & 1.8 & $A O$ & 110 & 8 & 5 & 6 & 400 & 18 & AO & 110 & 5 & 5 \\
\hline 7 & 400 & 4.5 & A & 80 & 10 & 12 & 7 & 400 & 4.5 & A & 80 & 10 & 8 & 7 & 400 & 4.5 & A & 80 & 2 & 4 \\
\hline 8 & 400 & 4.5 & A & 110 & 14 & 8 & 8 & 400 & 4.5 & A & 110 & 9 & 6 & 8 & 400 & 4.5 & A & 110 & 4 & 3 \\
\hline 9 & 450 & 1.8 & $A O$ & 80 & 12 & 13 & 9 & 450 & 1.8 & AO & 80 & 7 & 10 & 9 & 450 & 1.8 & $A O$ & 80 & 3 & 5 \\
\hline 10 & 450 & 1.8 & $A O$ & 110 & 14 & 10 & 10 & 450 & 1.8 & $A O$ & 110 & 6 & 8 & 10 & 450 & 1.8 & $A O$ & 110 & 1 & 3 \\
\hline 11 & 450 & 4.5 & A & 80 & 9 & 14 & 11 & 450 & 4.5 & A & 80 & 8 & 10 & II & 450 & 4.5 & A & 80 & 2 & 5 \\
\hline 12 & 450 & 4.5 & A & 110 & 13 & 15 & 12 & 450 & 4.5 & A & 110 & 10 & 7 & 12 & 450 & 4.5 & A & 110 & 5 & 4 \\
\hline 13 & 450 & 1.8 & A & 80 & 12 & 11 & 13 & 450 & 1.8 & A & 80 & 4 & 6 & 13 & 450 & 1.8 & A & 80 & 4 & 4 \\
\hline 14 & 450 & 1.8 & $A$ & 110 & 13 & 10 & 14 & 450 & 1.8 & A & 110 & 5 & 6 & 14 & 450 & 18 & A & 110 & 5 & 2 \\
\hline 15 & 450 & 4.5 & $A O$ & 80 & 11 & 15 & 15 & 450 & 4.5 & AO & 80 & 7 & 8 & 15 & 450 & 4.5 & $A O$ & 80 & 2 & 4 \\
\hline 16 & 450 & 4.5 & $A O$ & 110 & 15 & 14 & 16 & 450 & 4.5 & $A O$ & 110 & 10 & 7 & 16 & 450 & 4.5 & $A O$ & 110 & 3 & 5 \\
\hline
\end{tabular}

Table 2. Input data of different parameters. 


\subsection{Analysis Of Defect By Taguch Technique.}

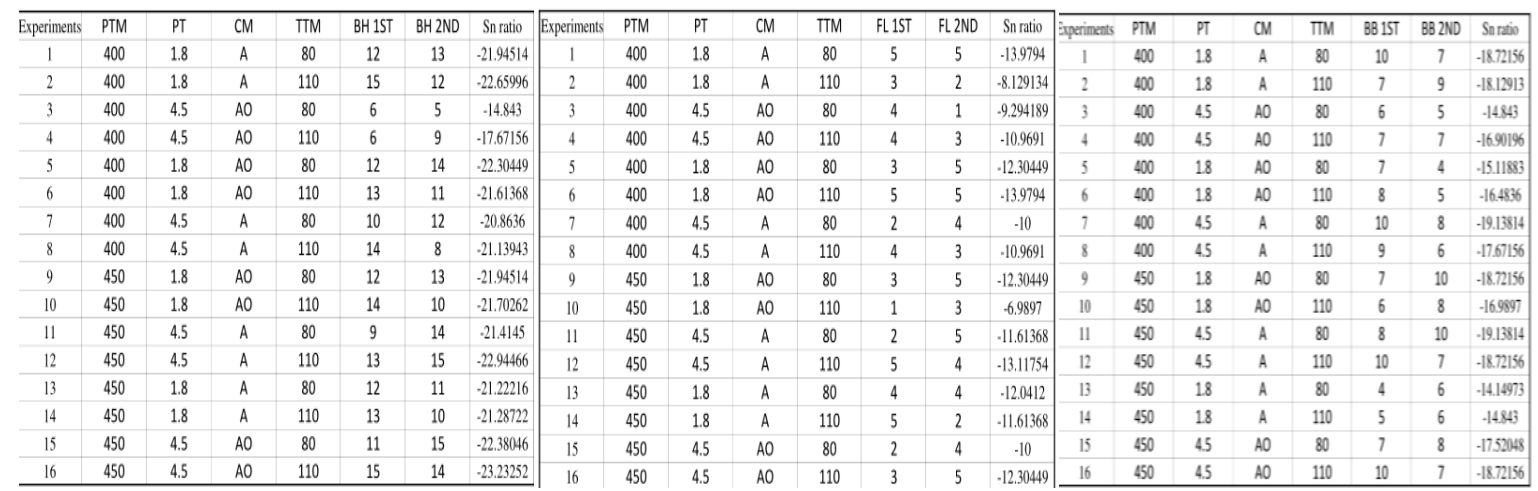

Table 3. Standard orthogonal L16 array with output results.

[PTM-Pouring temperature, PT- Pouring time, CM-Cooling medium, TTM-Tool temperature, BH-Blowhole, BB-Bubble, FL-Flash and SN- signal-to-noise ratio].

The experiments were conducted as per the standard orthogonal array. The selection of orthogonal array was based on the condition that the degree of freedom for the orthogonal array should be greater than or equal to sum of those defect parameters. In present investigation L16 orthogonal array was chosen. The defect parameter chosen are pouring temperature, pouring time, cooling and tool temperature .The experiments are consist of test and was assigned as shown in the table3. The experimental results are analyzed using Taguchi method and the significant parameters affecting defect have been identified as shown in the table4. Analysis are made using minitab-16 software in order to find statistical significance of various parameters like pouring temperature, pouring time, cooling medium and tool temperature on defect analysis of variance performed on the experimental data. From Taguchi defect response it is evident that poring temperature plays a significant role followed by pouring time, cooling medium and tool temperature in blowhole defect. Pouring time plays a vital role followed by cooling medium, pouring temperature and tool temperature in bubble defect. And finally tool temperature has large significant followed by cooling medium, pouring time and pouring temperature in flash defect.

\subsection{Response Table For Signal To Noise Ratios.}

Smaller is better

For Blowhole, Bubble and Flash.

\begin{tabular}{|c|c|c|c|c|}
\hline Level & Pouring temp & Pouring time & Cooling medium & Tool temp \\
\hline 1 & -20.38 & -20.84 & -21.68 & -20.86 \\
\hline 2 & -22.02 & -20.56 & -20.71 & -21.53 \\
\hline Delta & 1.64 & 1.27 & 0.97 & 0.67 \\
\hline Rank & 1 & 2 & 3 & 4 \\
\hline & & & & \\
\hline Level & Pouring temp & Pouring time & Cooling medium & Tool temp \\
\hline 1 & -17.13 & -16.64 & -17.56 & -17.17 \\
\hline 2 & -17.35 & -17.83 & -16.91 & -17.31 \\
\hline Delta & 0.22 & 1.19 & 0.65 & 0.14 \\
\hline Rank & 3 & 1 & 2 & 4 \\
\hline & & & & \\
\hline Level & Pouring temp & Pouring time & Cooling medium & Tool temp \\
\hline 1 & -11.2 & -11.42 & -11.43 & -11.44 \\
\hline 2 & -11.25 & -11.03 & -11.02 & -11.01 \\
\hline Delta & 0.04 & 0.38 & 0.41 & 0.43 \\
\hline Rank & 4 & 3 & 2 & 1 \\
\hline
\end{tabular}

Table 4. Defect response for signal to noise ratio. 
3.4 Main Effect Plots For Sn Ration.

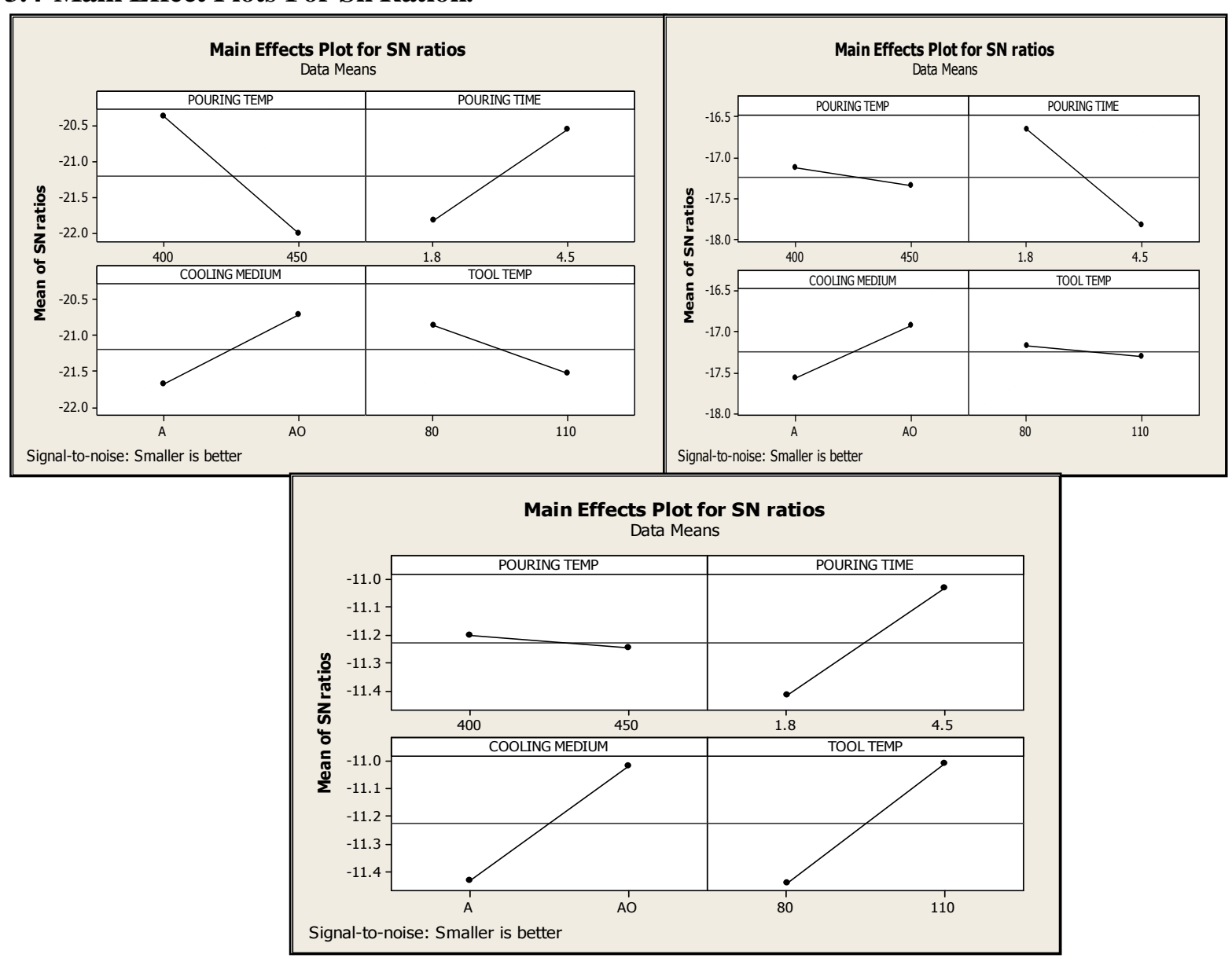

Figure 2. Main effect plot for SN ratio of Blowhole, bubble and flash defect.

\section{Conclusion.}

From defect test we can able to conclude that Pouring temperature plays a major role and pouring time, cooling medium and tool temperature plays a minor role in the blowhole defect when compared to other parameters as the temperature increases signal to noise ratio is being increased that influences the blowhole defect rate. In bubble defect pouring time and cooling medium plays a major role and pouring temperature and tool temperature plays a minor role, which can be seen in a graph as pouring time increases with increase in SN ratio thus concludes that pouring time plays a major role in bubble defect in a component. In flash defect tool temperature and cooling medium plays a vital role in flash defect response as tool temperature increases with increase in signal to noise ratio. And the other cooling and pouring time plays a minor role in flash defect response. Lastly we can able to conclude that the factors can be controlled in order to decrease the defect produced in a component.

\section{References.}

[1]. North American Die Casting Association (2009), 241 Holbrook Drive, Wheeling, IL 60090

[2]. Copyright $\odot 2009$ eFunda, Inc.

[3]. ASM Handbook Volume 15, Casting (2008 Edition).

[4]. http://www.diecasting.org/faq/ downloaded on 15 March , 2010

[5]. DYCOT Manuals of Foseco GmbH, Germany.

[6]. http://www.kenwalt.com/DiecastingAlloys.pdf downloaded on 15 March, 2010

[7]. http://www.custompartnet.com/wu/InjectionMolding downloaded on 15 March, 2010

[8]. Hallam C.P. and Griffiths W.D., (2004), "A model of interfacial heat transfer coefficient for the aluminium die casting process", Metallurgical and materials transactions B, vol.35B, pp. 723 .

[9]. Zhi-Peng a Guo, Shou-Mei a Xiong, Bai-cheng a Liu, Bai-cheng a Liu, Li b Mei, Allison b Jhon.,(2008)“Determination of the heat transfer coefficient at metal-die interface of high pressure die casting process of AM50 alloy" International journal of heat and mass transfer 51, pp. 6032-6038.

[10]. Zhi-Peng a Guo, Shou-Mei a Xiong, Bai-cheng a Liu, Bai-cheng a Liu, Li b Mei, Allison b Jhon. (2008), "Effect of process parameter, casting thickness and alloys on the interfacial heat transfer coefficient in the high pressure die casting process" Metallurgical and material transactions A, The minerals, metals and materials society and ASM international 2008. Vol.39A.pp. 2896. 
[11]. Zhi-peng GUO, Shoumei XIONG, SangHyun CHO and JeongKil CHOI.(2002), "Interfacial heat transfer coefficient between metal and die during high pressure die casting process of aluminium alloy" Journal of materials processing technology. Pp.130-131, 299303.

[12]. Pearsson a Anders, Hogmark b Sture, Bergstrm a Jens.(2004), "Temperature profile and condition for thermal fatigue cracking in brass die casting dies" Journal of materials processing technology 152.pp.228-236.

[13]. Micowski J.R and Teufert C.E.,(), "The control of impact pressure in the pressure die casting process", North America Die Casting Association. 81

[14]. Matthew S, Dargusch a, Dourb G, Schauer c, Dinnis C.M. and Savaged G (2006), "The influence of pressure during solidification of high pressure die cast aluminium telecommunication components" Journal of materials processing technology 180. Pp.37-43.

[15]. Chiang Ko-Ta, Liu Nun-Ming and Tsai Te-Chang. (2008) "Modelling and analysis of the effect of processing parameters on the performance characteristics in the high pressure die casting process of Al-Sl alloys". Int J Adv Manuf Technol 41:1076-1084, Springer-Verlag London.

[16]. "Final Report on Energy Consumption of Die Casting Operations", US department of energy grant/contract no. DE-FCo700ID13843.

[17]. Matthew Patrzalek, CRC CASTA/Prof. Ebrahim Shayan Dr. Dario Toncich Control of Cooling in Casting Dies, Based on Thermal Feedback

[18]. Yammagata H, Kasprzakb C., Aniolekb M, Kuritaa H and Sokolowskib J.H.(2008)“The effect of average cooling rates on the microstructure of $\mathrm{Al}-20 \% \mathrm{Si}$ high pressure die casting alloy used for monolithic cylinder blocks "Journal of materials processing technology 203.pp.333-341.

[19]. Ahuett Horacio, Garza R, Miller Allen.,(2003), "The effect of heat released during fill on the deflection of die casting "Journal of materials processing technology 142. Pp. 648-658.

[20]. Han Q. And Vishvanathan S., (2003) ,"Analysis of the mechanism of die soldering in aluminium die casting", Metallurgical and material transactions A, vol. 34A, pp.139.

[21]. Sumanth Shankar and Diran Apelian.,(2002)“Mechanism and preventive measures for die soldering during aluminium casting in a ferrous mould" JOM.

[22]. Hangi Yoshihiko and Utsunomiya Takao. (2009),"Fabrication of porous aluminium using gases intrinsically contained in aluminium alloy die casting" The minerals, metals and materials society and ASM international 2009, 1284-vol.40A, Metallurgical and material transactions A.

[23]. Sabau A.S. and Vishvanathan S.,(2002)“Micro porosity prediction in aluminium alloy castings" Metallurgical and materials transactions b Vol.33B.pp.243.82

[24]. Zhu J.D., Cockcroft S.L., and Maijer D.M.(2006)“Modelling of micro porosity formation in A 356 Aluminium Alloy Casting” Metallurgical and materials transactions A. Vol. 37A.pp.1075.

[25]. Zhua Hanliang, Guob Jingjie and Jiab Jun.,(2002), "Experimental study and theoretical analysis on die soldering in aluminium die casting" Journal of materials processing technology 123. Pp.229-235 